\title{
Evaluación del profesorado. Evaluación de la calidad docente
}

José Carreras Barnés

\section{Evaluación de la calidad docente del profesorado como componente del sistema de garantía interna de calidad de las universidades}

En el ámbito del Espacio Europeo de Educación Superior (EEES), la evaluación de la calidad docente del profesorado es un proceso que deben llevar a cabo fundamentalmente las propias universidades como elemento del sistema de garantía interna de calidad desarrollado en el marco del sistema europeo de calidad. A tal efecto, siguiendo el mandato de la conferencia ministerial de Berlín del año 2003, la ENQA, en cooperación con la EUA, la EURASHE y la ESIB, propuso los criterios y directrices para la garantía de calidad en el EEES [1], que fueron adoptados por la conferencia ministerial de Bergen del año 2005. De acuerdo con los mismos, las instituciones de educación superior deben disponer de medios que garanticen que el personal docente esté capacitado y sea competente para su trabajo. Deben disponer de procedimientos de incorporación del profesorado que incluyan medios para verificar que las personas seleccionadas poseen un nivel mínimo de competencia. Deben proporcionar oportunidades a los profesores de bajo rendimiento para que mejoren sus habilidades de forma que alcancen un nivel aceptable, y deben disponer de los medios que permitan darles de baja de sus funciones docentes si continúan siendo ineficaces.

Siguiendo los acuerdos de las conferencia ministeriales y dado que la Ley Orgánica de Modificación de la LOU (LOMLOU) [2] determinó que la garantía de calidad era un fin esencial de la política universitaria, la ANECA, la AQU y la ACSUG, a partir del año 2007, desarrollaron de forma conjunta el programa AUDIT [3], dirigido a orientar y apoyar a las universidades y a los centros universitarios en el diseño de sistemas de garantía interna de calidad, integrando las actividades relacionadas con la garantía de la calidad de las enseñanzas que ya se venían desarrollando. Por lo que respecta a la calidad de la actividad docente, las directrices incluidas en AUDIT especificaron que las universidades y los centros debían contar con mecanismos que aseguraran que el acceso, la formación, la evaluación, la promoción y el reconocimiento de su personal académico y de apoyo a la docencia se realizase con las debidas garantías para que cumpliera con las funciones que le son propias.

Actualmente, la normativa universitaria vigente en España permite que las universidades sean responsables de evaluar la capacitación docente de los candidatos en los procesos de selección del profesorado y de evaluar periódicamente la calidad de la actividad docente que desarrolla el profesorado ya en ejercicio.

\section{Evaluación de la capacitación docente de los candidatos en los procesos de selección del profesorado}

El proceso de selección del profesorado funcionario de los cuerpos docentes (profesores titulares y catedráticos de universidad) tiene lugar en dos fases [2]: una fase de acreditación nacional realizada por la ANECA, que tiene por objeto asegurar un nivel mínimo, y los concursos de
Catedrático de Bioquímica y Biología Molecular. Facultad de Medicina. Universitat de Barcelona.

E-mail

jcarreras@ub.edu 
acceso a plazas específicas realizados por las respectivas universidades, dirigidos a seleccionar los candidatos más adecuados de acuerdo con los perfiles de las plazas convocadas.

El proceso de acreditación nacional, desarrollado por la ANECA mediante el programa ACADEMIA [4], en el caso de acreditación para profesor titular, comporta la evaluación de cuatro dimensiones fundamentales: la formación, la experiencia investigadora, la experiencia docente o profesional y la experiencia en gestión y administración. El Real Decreto que regula este proceso [5] establece la tipología a utilizar para clasificar las aportaciones de los solicitantes, así como la puntuación asignada a cada una de las cuatro dimensiones: 5, 50, 40 y 5 puntos, respectivamente, sobre un total de 100 puntos. Además, determina que para obtener evaluación positiva es necesario obtener un total mínimo de 65 puntos y alcanzar como mínimo 60 puntos entre las dimensiones 'experiencia investigadora' y 'experiencia docente o profesional'. En la acreditación para catedrático de universidad, no se evalúa la dimensión 'formación' y la puntuación máxima asignada a las otras tres dimensiones es 55, 35 y 10 puntos, respectivamente. Para obtener evaluación positiva debe lograrse un total mínimo de 80 puntos y un mínimo de 20 puntos en la dimensión 'experiencia docente o profesional'. Por ello, las dimensiones 'experiencia investigadora' y 'experiencia docente o profesional' son decisivas, pero dado que sólo pueden perderse 20 puntos, la dimensión 'experiencia en gestión y administración' puede ser también condicionante del resultado. La normativa ministerial fija los mismos cinco criterios para evaluar la dimensión 'experiencia docente o profesional' en la acreditación para profesor titular y para catedrático: dedicación docente, calidad de la actividad docente, calidad de la formación docente, calidad y dedicación a actividades profesionales en empresas, instituciones, organismos públicos de investigación u hospitales, distintas a las docentes e investigadoras, y otros méritos relacionados con la actividad profesional. Pero la ANECA [4] estableció, a título orientativo, distintas puntuaciones máximas para los criterios mencionados, según que la acreditación fuera para profesor titular o para catedrático. En el primer caso, dichas puntuaciones fueron: 26,8 , 3 , 3 y 2 (adicionales) puntos, respectivamente, sobre un total de 40 puntos. En el segundo caso, sobre un total de 35 puntos, fueron: 22, 7, 3, 3 y 2 (adicionales) puntos.

Por lo que respecta a los concursos de acceso a plazas de los cuerpos docentes universitarios, la LOMLOU [2] determina que serán regulados por las propias universidades, de acuerdo con lo que establezcan sus estatutos. La normativa ministerial que desarrolla este tema [6] se limita a establecer que el procedimiento de dichos concursos debe valorar, en todo caso, el historial académico, docente e investigador del candidato, y su proyecto docente e investigador; así como contrastar sus capacidades para la exposición y debate en la correspondiente materia o especialidad, ante la comisión y en sesión pública. En general, los concursos desarrollados por las universidades constan de dos o tres pruebas (raramente una sola), que incluyen, en todos los casos, la exposición oral del currículo, del proyecto docente, del proyecto de investigación y, en el caso de plazas vinculadas, del proyecto asistencial sanitario, seguido de un debate con los miembros de la comisión. En los concursos a plazas de profesor titular (no en los concursos a plazas de catedrático), las pruebas generalmente incluyen, también, la exposición de un tema de la materia (libremente elegido por el candidato o elegido por el candidato entre tres resultantes de sorteo). Y los concursos a plazas de catedrático (raramente los concursos a plazas de profesor titular) incorporan la exposición y debate de un trabajo de investigación original [7]. ¿Son estos procedimientos adecuados para evaluar las competencias docentes de los concursantes? Depende, en gran parte, de los criterios de evaluación y de los baremos que se utilicen, los cuales son fijados por las comisiones que resuelven los concursos.

En cuanto a la contratación como profesor contratado doctor, definida como de carácter permanente por la LOMLOU, esta ley establece como requisito necesario la previa evaluación positiva por la ANECA o por los órganos de evaluación que la ley de las comunidades autónomas determine en el ámbito de sus respectivas competencias. Las normas del proceso de evaluación desarrollado por la ANECA [8] fijan cuatro criterios de evaluación: experiencia investigadora, experiencia docente, formación académica y experiencia profesional, y otros méritos. Dichas normas asignan como puntuaciones máximas para estos cuatro criterios $60,30,8$ y 2 puntos, respectivamente, y enumeran los méritos a con- 
siderar en cada criterio especificando las correspondientes puntuaciones máximas. Como requisitos mínimos para la evaluación positiva fijan alcanzar 50 puntos en la suma de los criterios 'experiencia investigadora' y 'experiencia docente', y 55 puntos en el total de los criterios.

La mayoría de las agencias de las comunidades autónomas que han desarrollado el proceso de evaluación previo a la contratación como profesor contratado doctor permanente [9] agruparon los méritos a evaluar en criterios semejantes a los establecidos por la ANECA, si bien los pesos relativos definidos para los diversos criterios fueron variables. Una situación especial se ha dado en Cataluña, por cuanto la Ley de Universidades de Cataluña, que estableció dos categorías dentro del profesor contratado doctor (la de profesor agregado y la de catedrático contratado), sólo exige la evaluación previa de la actividad investigadora como requisito imprescindible para poder participar en los concursos de acceso; la evaluación de la actividad docente se requiere solamente si así lo establecen las universidades en las correspondientes convocatorias.

Cabe señalar que, en el año 2006, la ANECA y las agencias autonómicas constituyeron la Red Española de Agencias de Calidad Universitaria (REACU) con el fin de promover su colaboración, y publicaron un documento sobre 'Armonización de la evaluación del profesorado' con recomendaciones consensuadas [9].

Respecto a los concursos de acceso a plazas de profesor contratado doctor permanente convocadas por las universidades, en general, se han desarrollado con procedimientos semejantes a los de los concursos a plazas de los cuerpos docentes [9].

\section{Evaluación de la calidad de la actividad docente}

La actividad docente es el conjunto de actuaciones que se realizan dentro y fuera del aula destinadas a favorecer el aprendizaje de los estudiantes con relación a los objetivos y competencias definidas en un plan de estudios y en un contexto institucional determinado. La responsabilidad de garantizar la calidad de la actividad docente que desarrolla el profesorado ya en ejercicio recae en cada universidad, y es ésta quien debe tener un sistema que asegure dicha calidad de forma fiable y sistemática. Las agencias de calidad colaboran con las universidades y tienen como función principal comprobar que los sistemas de calidad que éstas han diseñado se han establecido y se aplican de forma adecuada.

\section{Programa DOCENTIA}

El programa DOCENTIA [10] es un programa compartido por la ANECA y agencias de evaluación de comunidades autónomas, iniciado en el año 2008, con el objetivo de apoyar y facilitar la evaluación de la calidad de la actividad docente del profesorado que realizan las universidades, garantizando el rigor de la evaluación y su adecuación a los objetivos previstos. Se propone ayudar a las universidades a disponer de un modelo validado que sea apropiado, útil, viable y preciso, y constituir una herramienta para favorecer la cultura de la calidad, alineando la evaluación de la actividad docente con los criterios de garantía de calidad de las titulaciones universitarias, así como la actividad docente del profesorado con los objetivos de la institución.

El modelo en el que se fundamenta este programa establece como 'dimensión cero' o 'condición previa' la dedicación docente, de forma que si el profesor no desarrolla las actuaciones que la describen no procede determinar la calidad de la docencia. Y considera tres dimensiones como objeto de evaluación de la actividad docente:

- Planificación de la docencia: organización y coordinación docentes, resultados de aprendizaje previstos, actividades de aprendizaje previstas, criterios y métodos de evaluación, materiales y recursos docentes.

- Desarrollo de la enseñanza: actividades de enseñanza/aprendizaje realizadas, procedimientos de evaluación aplicados.

- Resultados: objetivos formativos logrados por los estudiantes, mejoras de la actividad docente conseguidas.

Por otra parte, el modelo DOCENTIA establece cuatro criterios de evaluación de la actividad docente, alineados con las dimensiones anteriores:

- Adecuación a los requerimientos establecidos por la universidad y el centro.

- Satisfacción de los demás agentes implicados en la enseñanza. 
- Eficiencia en el desarrollo en los estudiantes de las competencias establecidas.

- Orientación a la innovación docente.

Finalmente, el modelo considera que las fuentes y procedimientos de recogida de información seleccionados para la evaluación de la actividad docente deben tener cuatro características: evitar posibles sesgos en la información recogida, ser válidos, ser viables y permitir la interacción con los evaluados. De acuerdo con ello, y teniendo en cuenta las dimensiones a evaluar, propone el autoinforme del profesor, el informe de los responsables académicos y las encuestas a los estudiantes, reconociendo que las universidades pueden incorporar a su diseño otras fuentes y procedimientos de recogida de información de elaboración propia.

Atendiendo a estos principios, las universidades que decidan participar en el programa DOCENTIA, formalizándolo mediante un convenio con la ANECA y la correspondiente agencia de evaluación autonómica, deberán elaborar un documento o manual para la evaluación de la actividad docente de su profesorado que recoja, como mínimo, los aspectos siguientes:

- Aspectos estratégicos de la evaluación docente: finalidad, ámbito de aplicación, obligatoriedad, periodicidad, plan de difusión, agentes implicados.

- Dimensiones y criterios de evaluación.

- Fuentes y procedimientos de recogida de información: con inclusión de los formatos del autoinforme, del informe de las autoridades académicas y de la encuesta a los estudiantes.

- Procedimiento para realizar la evaluación: comités de evaluación, protocolo de evaluación, informe de evaluación, presentación de alegaciones.

- Procedimiento para la toma de acciones derivadas de la evaluación y para el seguimiento de éstas.

- Procedimiento para la difusión de los resultados.

Los manuales de evaluación docente elaborados por las universidades son verificados por una comisión de evaluación constituida por seis personas: el presidente (un académico), el secretario (un técnico de agencia de evaluación) y cuatro vocales (dos académicos, un profesional no académico y un estudiante). Estas personas son seleccionadas conjuntamente por la ANECA y las agencias de evaluación de las comunidades autónomas que participan en el programa, garantizando que no existan conflictos de intereses entre los evaluadores y las universidades cuyos manuales son objeto de verificación.

La comisión de evaluación, utilizando los protocolos elaborados por las agencias de evaluación, analiza los manuales de manera colegiada y emite un informe provisional de verificación que recoge las valoraciones, recomendaciones y propuestas de mejora, y que puede ser positivo, positivo con condiciones o negativo. La agencia de evaluación correspondiente remite estos informes a las universidades, que en el caso de recibir una valoración positiva con condiciones dispondrán de un plazo de seis meses para presentar las observaciones pertinentes. La comisión de evaluación revisa las modificaciones introducidas en los diseños originales y emite el informe definitivo de verificación.

Si el informe ha sido positivo, la universidad inicia las actuaciones previstas en su manual, pudiendo contar con el apoyo de la ANECA o de las agencias de evaluación autonómicas. El procedimiento diseñado se aplicará al menos durante dos años con carácter experimental y durante ese periodo será objeto de seguimiento por parte de la propia universidad y de las agencias de evaluación (que crearán al efecto una comisión de seguimiento), a fin de comprobar que la evaluación de la actividad docente se está llevando a cabo de acuerdo con el proyecto inicial, e introducir ajustes y mejoras atendiendo a las exigencias y necesidades del contexto de aplicación. El proceso de seguimiento implicará la elaboración anual de un informe de implantación por parte de las universidad, que será revisado por la comisión de seguimiento, la cual emitirá un informe señalando las posibles deficiencias detectadas y sugiriendo recomendaciones para mejorar la implantación del diseño. Valorado el segundo informe de implantación elaborado por la universidad, la comisión de seguimiento decidirá si el diseño puede ser certificado (para un período de cuatro años) o si debe aplicarse experimentalmente durante un año más.

Los resultados del desarrollo del programa DOCENTIA a finales del mes de abril del presente año eran los siguientes: se había resuelto el proceso de verificación de 54 manuales, 9 se hallaban en fase de alegaciones y 19 en fase de seguimiento. No habían presentado su diseño 
cinco de las universidades y otras cinco no habían participado en el programa [10].

El seguimiento de la aplicación del manual de evaluación docente de seis universidades, en el que ha participado este ponente, ha revelado que uno de los puntos débiles que suele presentarse es la baja capacidad de discriminación, debido principalmente a que el diseño no distingue de manera adecuada el mero cumplimiento de lo que constituyen las obligaciones docentes de todo profesor de aquellas actuaciones que revelan una actividad docente innovadora, creativa y generosa, y a que son escasos los niveles de evaluación establecidos. Se considera fundamental corregir este defecto; su persistencia conduciría al desprestigio del proceso y sería un importante factor de desmotivación del profesorado por lo que se refiere a la mejora de la calidad docente.

\section{Bibliografía}

1. European Association for Quality Assurance in Higher Education. Criterios y directrices para la garantía de calidad en el Espacio Europeo de Educación Superior. URL: http://www.enqa.eu/files/ESG\%20version\%20ESP.pdf.

2. Ley Orgánica 4/2007, de 12 de abril, por la que se modifica la Ley Orgánica 6/2001, de 21 de diciembre, de Universidades. BOE de 13-IV-2007. p. 16241-60.

3. ANECA. Programa AUDIT. URL: http://www.aneca.es/ actividadesevaluacion/evaluacionenseñanzas/audit. aspx.
4. ANECA.Programa ACADEMIA.URL:http://www.aneca. es/actividadesevaluacion/evaluacionprofesorado/acreditacion_nacional.aspx.

5. Ministerio de Educación y Ciencia. Real Decreto 1312/2007, de 5 de octubre, por el que se establece la acreditación nacional para el acceso a los cuerpos docentes universitarios. BOE de 6-X-2007. p. 40653-9.

6. Ministerio de Educación y Ciencia. Real Decreto 1313/2007, de 5 de octubre, por el que se regula el régimen de los concursos de acceso a cuerpos docentes universitarios. BOE de 8-X-2007. p. 40758-61.

7. Carreras J. Evaluación de la calidad docente y promoción del profesorado (VI). Legislación universitaria española (d): modificación de la Ley Orgánica de Universidades. Profesorado funcionario: de la habilitación a la acreditación (2004-2008) (2. ${ }^{\text {a }}$ parte). Educ Med 2009; 12: 131-47.

8. Resolución de 18 de febrero de 2005 , de la Dirección General de Universidades, por la que se modifican determinados aspectos del procedimiento de presentación de solicitudes de evaluación o informe de la Agencia Nacional de Evaluación de la Calidad y Acreditación para la contratación de personal docente e investigador, así como de los criterios de evaluación, establecidos en las resoluciones de 17 de octubre de 2002 y de 24 de junio de 2003, de la Dirección General de Universidades. BOE de 4-III-2005. p. 7875-87.

9. Carreras J. Evaluación de la calidad docente y promoción del profesorado (VII). Legislación universitaria española (e): modificación de la Ley Orgánica de Universidades. Profesorado contratado permanente (2004-2008). Educ Med 2010; 13: 205-21.

10. ANECA.ProgramaDOCENTIA.URL: http://www.aneca. es/actividadesevaluacion/evaluacionenseñanzas/docentia.aspx. 